\title{
Guest Editors Foreword
}

\author{
Leizhen Cai ${ }^{1}$ • Siu-Wing Cheng ${ }^{2}$ • Tak-Wah Lam ${ }^{3}$
}

Received: 20 August 2015 / Accepted: 4 September 2015 / Published online: 30 September 2015 (C) Springer Science+Business Media New York 2015

This special issue contains the extended journal versions of six selected papers that were presented at the 24th International Symposium on Algorithms and Computation (ISAAC), December 16-18, 2013 in Hong Kong. The International Symposium on Algorithms and Computation is an established theoretical computer science conference where high-quality papers in theoretical computer science are presented. The six articles in this issue were invited, submitted, and then reviewed thoroughly according to the usual, high standard of the journal.

Arge and Thorup consider the problem of designing a sorting algorithm that uses asymptotically fewest number of I/O transfers as well as asymptotically minimum internal computational time. Such a model captures both the I/O complexity of accessing external storages and the CPU processing time, which are the two most prominent computational resources in any computational problem. Arge and Thorup propose a new sorting algorithm and obtain lower bounds to show that their sorting algorithm is optimal in certain cases. Moreover, they design a priority queue structure that is both I/O- and RAM-efficient.

Farach-Colton and Tsai present an algorithm to sample a binomial distribution which is an important subroutine in many large-scale numerical simulations. Existing implementations for such sampling task run in time linear in number of binomial trials and/or suffer from precision loss. Farach-Colton and Tsai present the first algorithm that can generate a sample from a binomial distributione exactly in constant time with high probability, after some appropriate preprocessing.

\footnotetext{
Tak-Wah Lam

twlam@cs.hku.hk

1 The Chinese University of Hong Kong, Shatin, Hong Kong

2 University of Science \& Technology, Clear Water Bay, Hong Kong

3 University of Hong Kong, Pokfulam, Hong Kong
} 
A Bloom filter is a method for storing a set with reduced memory by allowing a small error probability. Naor and Yogev conduct a theoretical analysis of the Bloom filter in streaming setting and handle membership queries regarding the set of the last nelements. Naor and They give a data structure with low space to achieve, with high probability, constant query time and update time in the worst case.

Cheong, Har-Peled, Kim, and Kim consider a graph theoretic question that is motivated by graph drawing. A $k$-fan is a set of kedges that are incident to a common vertex, and a $k$-fan crossing refers to the situation that another edge crosses all these $k$ edges. Cheong et al. obtain improved upper bound on the number of edges in a graph that is free of any $k$-fan crossing. They also generalize the upper bound on the number of edges to graphs that satisfy some monotone graph property.

Van Bevern, Downey, Fellows, Gaspers, and Rosamond use an analog of the Myhill-Nerode methods to derive two results for hypergraphs. First, testing whether a hypergraph has cutwidth at most $k$ for some constant $k$ can be done in linear time. Second, it is impossible to express in monadic second-order logic whether a hypergraph has bounded (fractional, generalized) hypertree width.

Klein and Suri extend the pursuit-evasion problem to the domain of closed polyhedral surfaces. The problem is to bound the number of point pursuers needed to capture a point evader in the discrete time model. The authors prove that four pursuers always suffice and three pursuers are sometimes necessary for surfaces of genus zero. Then, they show that $4 \mathrm{~g}+4$ pursuers always suffice for surfaces of genus $g$. Furthermore, their results can be generalized to the case of weighted regions.

These six interesting articles cover a wide spectrum of topics, and demonstrate the breadth and depth of the research represented by the ISAAC. We thank all authors for submitting their articles to this special issue, and all referees for their time and efforts. We would also like to thank the editor-in-chief Ming-Yang Kao for supporting ISAAC and making this special issue possible.

Hong Kong, 2015 